\title{
Percepción de pasantes de medicina en servicio social sobre su plan de estudios
}

\author{
Perception of graduates of the school of medicine in social service on its \\ curriculum
}

Juan Manuel Muñoz Cano

Juan Antonio Córdova Hernández*

\section{RESUMEN}

Introducción: Los estudios de egresados proporcionan información para reorganizar el curriculum de las carreras. Con ese objetivo se aplicó un cuestionario a pasantes de medicina en servicio social, que se desarrolla una vez terminado los créditos y el año de internado. Métodos: Se elaboró un cuestionario que explora opinión acerca del desarrollo de los cursos, papel de las asignaturas, formación hacia el servicio social, recomendaciones para mejorar el curriculum. Se aplicó online en noviembre de 2016 y respondieron 24 médicos en servicio social. Resultados: Se identificaron cuatro áreas de oportunidad. Persistencia de métodos de enseñanza tradicionales. Necesidad de modificar el énfasis en el enciclopedismo. Necesidad de fortalecer la educación clínica. Introducir las competencias generales de las asignaturas comunes en los programas de asignatura de la carrera. Conclusiones: La opinión de los egresados plantean la necesidad de comenzar una reorientación del énfasis en la enseñanza de la medicina.

Palabras Clave: Curriculum tradicional; aprendizaje autodirigido; educación médica.

\section{ABSTRACT}

Introduction: Graduates studies provide information for reorganizing the curriculum of races. For that purpose a questionnaire was applied interns of medicine in social service, which develops once completed credits and year of boarding school. Methods: A questionnaire that explores opinion about the development of them courses, role of the subjects, training toward the service social, recommendations to improve the curriculum, was developed. It was applied online 2016 November and they responded 24 doctors in social service. Results: We identified four areas of opportunity. Persistence of traditional teaching methods. Need of change the emphasis in the encyclopedic. Need to strengthen the clinical education. Delete General Training Area subjects. Conclutions: The opinions of them graduates pose the need of begin a reorientation of the emphasis in medical education.

Keywords: Traditional curriculum; learning; medical education.

* División Académica de Ciencias de la Salud, Universidad Juárez Autónoma de Tabasco

\section{INTRODUCCIÓN}

Las encuestas de opinión son instrumentos para reconsiderar los procesos educativos y forman parte de los estudios que se hacen a egresados. La relevancia de estos estudios retrospectivos para la educación médica, es que proporcionan información para evaluar las causas del desempeño de los egresados, analizada en función de su integración al ambiente de trabajo (Guzmán y otros, 2008).

Los estudios de egresados se encuentran en los planes transversales de la Universidad Juárez Autónoma de Tabasco (UJAT) y tienen al menos dos propósitos fundamentales. Uno es contar con la percepción de los egresados para retroalimentar la reestructuración de planes y programas de estudio. La segunda es atender las indicaciones de los organismos acreditadores, como es el caso del Consejo Mexicano de Acreditación de la Educación Médica (COMAEM) para médico cirujano.

De acuerdo con la Estrategia propuesta en el Plan de Desarrollo de la División Académica de Ciencias de la Salud (DACS) de la UJAT, estos estudios deben considerar no sólo la función transversal de la Universidad sino los de la licenciatura de médico cirujano en particular. Los planes de estudio (contenidos y métodos de evaluación) han de estar en coherencia con el perfil de egreso, la misión y la visión de las 
Perspectivas Docentes 63

Percepción de pasantes de medicina en servicio social sobre su plan de estudios

Perception of graduates of the school of medicine in social service on its curriculum

Juan Manuel Muñoz Cano / Juan Antonio Córdova Hernández.

licenciaturas. Esto se ha de lograr mediante la mejora continua del plan de estudios (Jiménez, 2014, p. 71).

\section{ENCUESTAS DE EGRESADOS PARA LA REESTRUCTURACIÓN CURRICULAR}

Son instrumentos que deberían proporcionar información para la reestructuración curricular que periódicamente se realiza para mantener la pertinencia del plan de estudios. Se entiende que: "debemos ser conscientes de que los planteamientos teóricos y nuestra capacidad de análisis no son suficientes para anticiparnos a los cambios y a los problemas que éstos generan y de que no tenemos capacidad operativa real para resolverlos" (Gual y Fundación Educación Médica [FEM], 2012, p. 20). Las encuestas dirigidas a esta causa se consideran evaluaciones verdaderas sólo si se emplean en la toma de decisiones en el diseño curricular (Guzmán y otros, 2008).

Por ello la reestructuración curricular debe centrarse en nuevos paradigmas que no sólo deben ser claros para los diseñadores del curriculum sino también plantearse como necesidades de los egresados que inciden en los cambios curriculares. "Los contenidos curriculares tradicionales no bastan para el médico del futuro y nos deben estimular en la búsqueda prospectiva de otras áreas que debemos incorporar en la formación médica" (Gual y FEM, 2012, p. 36), entre ellas las centradas a las buenas prácticas en el desempeño con los pacientes y esas capacidades deben ser lo más relevante en los procesos de evaluación de los procesos educativos (General Medical Council [GMC], 2009, p. 59).

\section{ENCUESTAS DE EGRESADOS PARA ATENDER INDICADORES DE ORGANISMOS ACREDITADORES}

En esta categoría se encuentran los cuestionarios de la Asociación Nacional de Universidades e Instituciones de Educación Superior (ANUIES). Estos cuestionarios se aplican a egresados de cohortes con cinco años de haber finalizado sus estudios (ANUIES, s.f.) y para médico cirujano de la UJAT se tienen dos estudios de egresados y dos de empleadores. Los de la cohorte de egresados 1997 dado a conocer en 2004, y el de la de 2002 publicada en 2012 (UJAT, 2004; 2006; 2007; 2012). Como se entiende, tienen escasa relevancia para la reestructuración curricular ${ }^{1} \mathrm{y}$ se consideran pseudoevaluaciones (Guzmán y otros, 2008). En el caso del indicador 47 del COMAEM: "El seguimiento de egresados es congruente con el perfil profesional de la escuela o facultad, verifica el rendimiento académico y profesional del egresado; y realimenta el desarrollo del plan de estudios". Para COMAEM se requiere además de la reestructuración del plan de estudios, mejorar el programa de seguimiento de egresados (Jiménez, 2014, p. 46).

Este proceso de mejora tiene como propósito que dicho programa sirva efectivamente para atender necesidades de los estudiantes a su egreso (aumentar el número de quienes pueden obtener el título mediante la aprobación del Examen General de Egreso del Centro Nacional de Evaluación de la Educación Superior (EGEL-CENEVAL), pues quienes presentan este examen no obtienen el puntaje mínimo para ser considerados en el nivel de suficiencia. Esto contrasta con el logro de suficiente y sobresaliente de otras universidades (Guerrero et al., 2015).

\section{EL PROGRAMA DE ESTUDIOS DE MÉDICO CIRUJANO DE LA UJAT}

Desde 1958 y hasta 2006 el plan de estudios fue esencialmente el mismo cuando se intentó transitar a la flexibilidad (Muñoz y otros, 2007). El plan de estudios vigente data de 2010. Como los anteriores, presenta la separación de los contenidos en un bloque de asignaturas denominadas básicas, y otro de clínicas. Esto es producto del esquema de Flexner de principios del siglo anterior, el cual fue creado para homogeneizar la enseñanza de la medicina en Estados Unidos. Muestra la preocupación por insertar conceptos de la ciencia de finales del siglo XIX para mejorar una práctica de la medicina que distaba de ser óptima para el estado del conocimiento de la época. Sin embargo, al no tener sustento en teorías de aprendizaje un siglo después se ha convertido en un obstáculo para la integración de la ciencia a la clínica (Irby, Cooke y O’Brien, 2010).

La misión del programa educativo se centra en dos aspectos fundamentales. La construcción de competencias profesionales que debe estar explícita en el desempeño y la capacidad del aprendizaje autodirigido y permanente ${ }^{2}$ :

Formar médicos generales de calidad que contribuyan de manera significativa a la prevención y atención de los problemas de salud del individuo y de la sociedad, a través de una formación sólida e integral, capaces de aprender de 
Perspectivas Docentes 63

Percepción de pasantes de medicina en servicio social sobre su plan de estudios

Perception of graduates of the school of medicine in social service on its curriculum

Juan Manuel Muñoz Cano / Juan Antonio Córdova Hernández.

manera permanente y autónoma, con alto sentido humanístico, científico, tecnológico y ético (UJAT, 2010).

Los programas educativos en la UJAT cuentan con asignaturas comunes. Esta tendencia se inició en 1985 como parte del "Proyecto de excelencia y superación académica" que dispuso que para la DACS bioquímica y anatomía se insertaran en los programas de enfermería y odontología para "optimizar los recursos materiales, económicos y administrativos" (Romero, 2005, p. 76).

Posteriormente se modificaron los programas y se insertaron para toda la universidad asignaturas del área general, como remediales debido a la pobre calidad del sistema educativo medio superior. Estas asignaturas eran hasta 2016 Cultura ambiental, Metodología, Ética, Filosofía, Lengua extranjera, Lectura y redacción, Derechos humanos, Pensamiento matemático y Herramientas de computación. Sin embargo, la estructura de los programas de estas asignaturas fueron hechos con objetivos pobremente elaborados por lo que no hay coherencia entre los contenidos ni de manera interna ni con los programas (Pérez, Pérez y García, 2016; Pérez, Pérez y Méndez, 2016).

\section{OBJETIVO}

Categorizar las percepciones de los egresados ${ }^{3}$ en el momento de pasar a un escenario de práctica profesional sin supervisión para retroalimentar la reestructuración curricular desde la perspectiva del pasante en servicio social. Las percepciones se analizarán en función de los dos ejes principales de la misión: a) resolver problemas de salud de la población donde se desempeñan, b) tener capacidades para el aprendizaje autodirigido.

\section{MATERIAL Y MÉTODO}

\section{TIPO DE ESTUDIO}

Se trata de una investigación descriptiva, analítica, transversal y retrospectiva ya que analiza las causas del descontento o la satisfacción de los egresados respecto a su desempeño en un ambiente de la vida real.

\section{RECOLECCIÓN DE DATOS}

Para explorar las opiniones de los pasantes en servicio social se construyó en la DACS-UJAT un cuestionario para explorar a) La opinión acerca del desarrollo de los cursos, b) Opinión acerca de conocimientos y habilidades aprendidos, c) Opinión acerca de la formación hacia el servicio social, d) Recomendaciones para mejorar el perfil de formación profesional. El cuestionario siguió recomendaciones de Guzmán y otros (2008).

En junio de 2015 se realizó una prueba piloto. Al revisar las respuestas se hizo necesario modificar preguntas para que se lograra obtener un panorama más objetivo de las percepciones de los estudiantes. En noviembre de 2016 se aplicó el cuestionario de percepción del proceso de educación médica a los 110 egresados que iniciaron el servicio social en julio de ese año. Para ello se envió a sus direcciones electrónicas una copia del mismo acompañado por una solicitud de la coordinación de la licenciatura. Se recibieron 24 respuestas (22\% del universo).

\section{CONSIDERACIONES ÉTICAS}

Los cuestionarios se manejaron de manera confidencial por lo que la identidad de los participantes se ocultó y se manejó mediante una clave.

\section{RESULTADOS}

En la primera sección, relativa a aspectos operativos, sólo uno de los pasantes $(4.2 \%)$ terminó la licenciatura en nueve semestres previo al año de internado y al de servicio social. También sólo uno suspendió durante un semestre su trayectoria escolar, por lo que también pudiera haber terminado en nueve semestres. Los estudiantes que respondieron tuvieron promedio de calificaciones mayor a nueve en su mayoría, $17(71 \%)$ y seis de ellos el promedio fue mayor de siete pero menor de nueve (25\%). Respondieron haber intentado obtener la mejor calificación posible (95\%).

Acerca de su licenciatura (pregunta 7) respondieron que trabajar mucho para aprobar fue nada o poco importante $46 \%$ lo que si fue muy importante para $25 \%$. Poder elegir las asignaturas fue nada importante para $25 \%$, poco $17 \%$, y regular $29 \%$. El enfoque en medicina general fue importante 
Perspectivas Docentes 63

Percepción de pasantes de medicina en servicio social sobre su plan de estudios

Perception of graduates of the school of medicine in social service on its curriculum

Juan Manuel Muñoz Cano / Juan Antonio Córdova Hernández.

mucho y muy importante para 50\%. Eligieron medicina por ser de prestigio académico como muy importante $25 \%$.

La promoción del aprendizaje en la mayor parte de las asignaturas fue primordialmente con base en exposiciones orales (67\%), elaboración de trabajos escritos (46\%), participación en proyectos de investigación $(37 \%)$ y prácticas clínicas y comunitarias (20\%). Estas cifras suman más de 100 porque se respondió más de un método por asignatura (Pregunta 8). Para los pasantes el proceso que más contribuyó a su formación fueron las prácticas clínicas y comunitarias $(72 \%)$ y el aprendizaje con base en proyectos o problemas (38\%); mencionaron los trabajos escritos $(25 \%)$ y las prácticas con inclusión de los simuladores (25\%) (Pregunta 9).

Las asignaturas consideradas de mayor importancia (pregunta 10) fueron las del área clínica (67\%) y las del área básica (29\%) y sólo $4 \%$ para actividades extracurriculares. Fuera quedaron el área general, prácticas profesionales y prácticas de laboratorio incluyendo disecciones.

El mayor énfasis en la evaluación para la calificación final (pregunta 11) estuvo en los exámenes escritos con preguntas de opción múltiple (46\%), exámenes escritos con preguntas abiertas $(25 \%)$, exámenes orales $(12.5 \%)$, exámenes prácticos $(12.5 \%)$ y ensayos (4\%). La resolución de casos o situaciones problema fue nada y poco importante (46\%).

Sobre los tres principales obstáculos que le impidieron terminar antes (pregunta 12), las asignaturas del tronco común (62\%), la estructura del plan de estudios (42\%), el límite de créditos $(42 \%)$ y la negativa de los tutores a permitirles inscribirse en más asignaturas (25\%). Algunos de ellos tuvieron problemas económicos y de tiempo (17\%). Aunque no por ser un obstáculo directamente se quejaron de la saturación de grupos (25\%).

A la pregunta 13, "Escribe las 3 asignaturas que consideras fueron las más difíciles de aprobar respondieron lo descrito en la tabla 1.

\begin{tabular}{|l|l|l|}
\hline & $f$ & $\%$ \\
\hline Neurología & 10 & 42 \\
\hline Cardiología & 9 & 38 \\
\hline
\end{tabular}

\begin{tabular}{|l|l|l|}
\hline Endocrinología & $\mathbf{7}$ & $\mathbf{2 9}$ \\
\hline Morfología & 5 & 21 \\
\hline Bioquímica & 4 & 17 \\
\hline Dermatología & 4 & 17 \\
\hline Microbiología y parasitología & 4 & 17 \\
\hline Anatomía patológica & 3 & 13 \\
\hline Inmunología & 2 & 8 \\
\hline Ginecología y obstetricia & 2 & 8 \\
\hline
\end{tabular}

Tabla 1. Asignaturas más difíciles de aprobar

A la pregunta 14 "Escribe las 3 asignaturas que más aportaron a tu competencia como médico" respondieron lo descrito en la tabla 2

\begin{tabular}{|l|l|l|}
\hline & $f$ & $\%$ \\
\hline Propedéutica & 14 & 58 \\
\hline Ginecología y obstetricia & 9 & 38 \\
\hline Pediatría & 9 & 38 \\
\hline Fisiología & 9 & 38 \\
\hline Morfología & 5 & 21 \\
\hline Fisiopatología & 5 & 21 \\
\hline Urgencias & 5 & 21 \\
\hline Farmacología & 4 & 17 \\
\hline Prácticas profesionales & 2 & 8 \\
\hline Salud pública & 2 & 8 \\
\hline Medicina interna & 2 & 8 \\
\hline
\end{tabular}

Tabla 2. Asignaturas que más aportaron a la educación médica

A la pregunta 15 "Si pudieras eliminar 3 asignaturas al plan de estudios, ¿cuáles serían?" respondieron lo descrito en la tabla 3.

La información para el desempeño en el servicio social (pregunta 16) fue sobre todo la que se proporcionó en la reunión informativa de la coordinación de la licenciatura, previa a la adscripción de las plazas (75\%). También habían recibido información de los estudiantes de otros ciclos escolares (50\%) y menos de sus profesores y tutores académicos (21\%). Para el desempeño (pregunta 17) consideraron como primordial el 
Perspectivas Docentes 63

Percepción de pasantes de medicina en servicio social sobre su plan de estudios

Perception of graduates of the school of medicine in social service on its curriculum

Juan Manuel Muñoz Cano / Juan Antonio Córdova Hernández.

manejo de las Normas y Guías de la Práctica Clínica (92\%) conocimiento de asignaturas clínicas (71\%), y primeros auxilios y asignaturas básicas (50\%). Los estudiantes no consideraron importante el manejo de información en línea, sólo $4 \%$ lo mencionó como necesario.

\begin{tabular}{|l|l|l|}
\hline & $f$ & $\%$ \\
\hline Pensamiento matemático & 15 & 63 \\
\hline Cultura ambiental & 14 & 58 \\
\hline Herramientas de computación & 11 & 46 \\
\hline Lectura y redacción & 7 & 29 \\
\hline Inglés & 5 & 21 \\
\hline Derechos humanos & 4 & 17 \\
\hline Filosofía & 3 & 13 \\
\hline Ética & 2 & 8 \\
\hline Bioética & 1 & 4 \\
\hline Salud mental & 1 & 4 \\
\hline Metodología de la investigación & 1 & 4 \\
\hline
\end{tabular}

Tabla 3. Asignaturas que deberían eliminarse

La principal preocupación de los pasantes (pregunta 18) fue educar en salud a la población (71\%), atender las enfermedades de la población (58\%), apoyar en las campañas de prevención de enfermedades no transmisibles (33\%), y contribuir a la vigilancia epidemiológica y desarrollar un proyecto de investigación (25\%). El principal obstáculo (pregunta 19) fue el llenado de formatos $(72 \%)$, el bajo salario (59\%), la falta de apoyo de la Secretaría (55\%), y las percepciones de la comunidad hacia las enfermedades (38\%) así como los problemas de las comunidades $(25 \%)$.

Los estudiantes calificaron el proceso formativo (pregunta 20) como excelente $(29 \%)$, bueno $(58 \%)$ y regular $(13 \%)$.

\section{DISCUSIÓN}

En las respuestas de esta muestra de pasantes a la percepción de un programa escolar elaborado de manera tradicional, con base en asignaturas y bloques, se tuvieron cuatro categorías de análisis. 1) La necesidad de superar los métodos y estrategias tradicionales. 2) La necesidad de disminuir la tendencia al verlo todo, agotar los temas de una asignatura. 3) La necesidad de ampliar las capacidades para la práctica de la medicina con base en disciplinas clínicas. 4) La necesidad de eliminar las asignaturas impuestas como remediales para las deficiencias del sistema de educación media superior.

\section{USO DE MÉTODOS Y ESTRATEGIAS INNOVADORES}

Los pasantes respondieron que los procesos de enseñanza se centran en la exposición oral (67\%), muchas veces elaborada por los estudiantes, quienes carecen de experticia en cualquier tema del currículum cuando lo hacen. Este es un área de oportunidad ya que de acuerdo a las tendencias, incluso en la UJAT, de transitar hacia la formación de capacidades que se evalúen mediante la ponderación del desempeño, se debería trabajar hacia el aprendizaje (UJAT, 2011, p.). También respondieron que el examen de tipo test es la principal herramienta para asignar calificaciones.

\section{ANALIZAR TEMAS A PROFUNDIDAD MÁS QUE AGOTAR EL TEMARIO}

Los pasantes hicieron énfasis en que algunas asignaturas resultaron difíciles de aprender debido a la inmensa cantidad de información contenida en el programa (Tabla 1). Por ejemplo, "por la cantidad de temas y porque en seis meses teníamos que acabar tres materias: morfología, histología, embriología" (1-M). Microbiología y parasitología "porque era una materia que me resulto difícil y tuve problemas para pasarla" (6-M).

En la escuela de medicina de la UJAT, como funciona en la mayor parte de las Universidades de América Latina sólo basta la repetición, la memorización acrítica persiste en el curriculum. Así, en una visión petrificada de la educación médica del siglo $\mathrm{XVII}$ :

Su ingreso a la facultad significó un cambio radical, por el tipo de materias y la profundidad y amplitud del conocimiento que se abordaba. La primera asignatura fue anatomía. (Sufrió) una conmoción cuando hojeó el tratado de anatomía humana de Testud y Latarjet, obra laureada por la Academia de Medicina de París en 1902, con bellas y precisas láminas, sabia conjunción 
Perspectivas Docentes 63

Percepción de pasantes de medicina en servicio social sobre su plan de estudios

Perception of graduates of the school of medicine in social service on its curriculum

Juan Manuel Muñoz Cano / Juan Antonio Córdova Hernández.

de arte y ciencia, convertida en alta disciplina del conocimiento médico (Ortiz, 2004: 161-162).

Esas asignaturas enciclopédicas no son percibidas de esa manera tan romántica por los estudiantes cuando terminan la escuela de medicina (Montemayor, 2006). Tan así que se les califica como "intrascendentes" una vez que se han desarrollado en el servicio (Watmough et al., 2009). La tendencia para integrar la ciencia al arte de la medicina, formar al "médico del mañana" en los sistemas educativos se dirige a considerar la evidencia científica, la experiencia clínica y los valores de los pacientes como un todo (GMC, 2009; Gregory y Armstrong., 2012).

\section{AUMENTAR EL ÉNFASIS EN LA EDUCACIÓN HACIA LA ATENCIÓN DE CALIDAD}

Los pasantes valoraron las asignaturas del área clínica como principal sustento a su formación. Propedéutica "es unas de las materias donde se explica relación médico-paciente, y principalmente la exploración general como se debe realizar" (1-M) y "tuvimos un excelente maestro" (9-H). Elevar los estándares académicos en el caso de medicina requiere de la comprensión de la complejidad de recursos para la elaboración de diagnósticos, así como el trabajo con base en ejes que integren contenidos conceptuales y procedimentales que se encuentran diseminados en diferentes disciplinas.

Para la mayoría de los modelos educativos, lo cual se refleja en las tablas de morbilidad y egresos, primero se hace el diagnóstico y posteriormente se fuerza a los datos clínicos a sustentarlo. Esto deriva de un catálogo limitado de enfermedades a las que deben corresponder los síntomas, por ejemplo, a cefalea el de tifoidea que se corrobora con la prueba inespecífica de las reacciones febriles (uso de una prueba de baja especificidad); a estornudos, fiebre y tos, infección de vías respiratorias que requiere antibióticos (se propicia la resistencia a los antibióticos).

Un proceso de mayor complejidad habría de comenzar por la lectura de los problemas de la comunidad. Ya que se hace con base en tablas de morbilidad, entre otros indicadores, sin pretender minimizar el trabajo que implica colectar datos y elaborar las tablas nacionales y locales, en esa lectura se hace necesaria la ponderación de las causas los años de vida saludable perdidos a causa de enfermedad y discapacidad.

\section{ELIMINACIÓN DE LAS NUEVE ASIGNATURAS DEL ÁREA GENERAL}

Los pasantes expresaron su descontento con las asignaturas del área general $(20 \%$ de los créditos de la licenciatura de médico cirujano) y su opinión acerca de que deberían eliminarse del plan de estudio (Tabla 3). Hubo consenso para "pensamiento matemático" (63\%), "cultura ambiental" (58\%), "herramientas de computación" (46\%), "lectura y redacción" $(29 \%)$, "inglés" (21\%), "derechos humanos" (17\%) y "filosofía" $(13 \%)$.

La limitada capacidad de estructuración y desarrollo de objetivos de aprendizaje de quienes realizaron los programas de estudio del área general de esas nueve asignaturas (Pérez, Pérez y García, 2016; Pérez, Pérez y Méndez, 2016) persistentes en el curriculum de todas las licenciaturas de la UJAT por la idea de optimizar recursos, podría explicar el rechazo de los pasantes en servicio social.

\section{CONCLUSIONES}

Los pasantes en servicio social manifestaron la necesidad de aumentar el énfasis en la educación médica hacia la educación de la población hacia el estilo de vida saludable y la atención de enfermedades. Consideraron primordial el manejo de Normas y Guías de la Práctica Clínica, en énfasis en el aprendizaje de las asignaturas clínicas así como primeros auxilios.

No se mencionó aumentar capacidades para aprendizaje en línea probablemente porque las actividades académicas tradicionales no se apoyan en las TIC. Esto significaría que no se forma para el aprendizaje autodirigido y autónomo, explícito en la misión de la licenciatura. 
Perspectivas Docentes 63

Percepción de pasantes de medicina en servicio social sobre su plan de estudios

Perception of graduates of the school of medicine in social service on its curriculum

Juan Manuel Muñoz Cano / Juan Antonio Córdova Hernández.

\section{NOTAS}

1. Los estudios de egresados que realiza la Universidad en concordancia con la ANUIES se aplican a quienes tienen cinco años de haber obtenido su documento de terminación de estudios, una vez finalizado su servicio social. Las preguntas relacionadas con la institución educativa no retroalimentan el curriculum (ANUIES, s.f.):

Satisfacción de los egresados en cuanto a la formación recibida por la universidad. Seleccionar entre excelente, buena, regular, insatisfactoria.

Opinión de los egresados en cuanto al clima universitario (instalaciones, capacidad y cumplimiento de profesores, limpieza, respeto a los derechos y obligaciones, equipamiento, laboratorio, bibliotecas, comportamiento de las autoridades, transparencia en el uso de los recursos citados). Seleccionar entre excelente, buena, regular, insatisfactoria.

2. Aprendizaje autodirigido. "Es el proceso por el cual los individuos toman la iniciativa, con o sin la ayuda de otros, de diagnosticar sus necesidades de aprendizaje, formular sus metas de aprendizaje, identificar los recursos humanos y materiales para aprender, elegir e implementar las estrategias de aprendizaje adecuadas y evaluar los resultados de su aprendizaje" (Parra y otros, 2010).

3. Para este estudio se considera como egresados a los pasantes en servicio social. En algunos estudios se les cataloga como recién egresados (Pontificia Universidad Javeriana, 2012). En el pasado reciente se les consideró estudiantes y se le confirieron créditos a esta etapa. Sin embargo, por fines administrativos (los estudiantes sólo pueden permanecer siete años inscritos en la UJAT) su estatus real no es de estudiantes ni de egresados.

\section{REFERENCIAS}

Anónimo. (2004). In memorian. Semblanza de un científico. Dr. Demetrio Sodi Pallares. Salud Pública de México, 46(1), 89-91. Recuperado de http://www.scielo.org.mx/scielo.php?script=sci arttext\&pid=S0036-36342004000100012\&lng=es.

Asociación Nacional de Universidades e Instituciones de Educación Superior. (S. F.). Guía para formular el Programa Integral de Fortalecimiento Institucional. 20122013. Recuperado de http://pifi.sep.gob.mx/ScPIFI/ GPIFI 2012 20012012/guia/5/4

General Medical Council. (2009). Tomorrow's Doctors. Education Outcomes and standards for undergraduate medical education. London: General Medical Council. Recuperado de $\quad$ http://www.gmc-uk.org/Tomorrow s Doctors 1214. pdf 48905759.pdf

Gregory, A. y Armstrong, R. (2012). Flaws in the fabric? Medicine Journal of Australia, 197(7), 361. doi: 10.5694/mja12. c1001

Gual Sala, A. y Fundación Educación Médica. (2012). Aprender a ser médico: responsabilidad social compartida. Madrid: Fundación Educación Médica. Recuperado de https://www. cgcom.es/sites/default/files/aprender ser medico/index.html

Guerrero-Ávila, J. J., Romero-González, J., y Noriega, R. (2015). Análisis de la competencia en medicina visto a través del EGEL-MG. Cultura Científica y Tecnológica, 49(10), 26-36. Recuperado de http://erevistas.uacj.mx/ojs/index.php/culcyt/ article/view/157/152

Guzmán Silva, S., Febles Álvarez-lcaza, M., Corredera Marmolejo, A., Flores Machado, P., Tuyub España, A., y Rodríguez Reynaga, P. A. (2008). Estudio de seguimiento de egresados: recomendaciones para su desarrollo Innovación Educativa, 8(42), 19-31. Recuperado de http://www.redalyc. org/pdf/1794/179421234003.pdf

Irby, D. M., Cooke, M., y O'Brien, B. C. (2010). Calls for reform of medical education by the Carnegie Foundation for the 
Perspectivas Docentes 63

Percepción de pasantes de medicina en servicio social sobre su plan de estudios

Perception of graduates of the school of medicine in social service on its curriculum

Juan Manuel Muñoz Cano / Juan Antonio Córdova Hernández.

Advancement of Teaching: 1910 and 2010. Academic Medicine, 85(2), 220-227. doi: 10.1097/ACM.0b013e3181c88449

Jiménez-Sastré, A. (2014). Plan de desarrollo divisional 20142018. Universidad Juárez Autónoma de Tabasco. Recuperado de http://www.ujat.mx/dacs/22768

Kalman, J. (2008). Discusiones conceptuales en el campo de la cultura escrita. Revista Iberoamericana de Educación, 46, 107134. Recuperado de http://www.rieoei.org/rie46a06.htm

Montemayor Flores, B. G. (2006). El significado de la práctica de disección para los estudiantes de medicina. International Journal of Morphology, 24(4), pp. 575-580. doi: 10.4067/S071795022006000500010

Muñoz-Cano, J. M., Maldonado-Salazar, T. D. N. J., Gómez, R. E., Melzer, J. A., Castillo-Orueta, M. L., y López-López, E. D. C. (2014). Reorientación del perfil de egreso de la licenciatura de médico cirujano. Horizonte Sanitario, 6(2), 9-17. Recuperado de http://revistas.ujat.mx/index.php/horizonte/article/view/218/158

Ortiz Quesada, F. (2004) Los viajes de Cordelia. México: latros.

Parra, P., Pérez, C., Ortiz, L., y Fasce, E. (2010). El aprendizaje autodirigido en el contexto de la educación médica. Revista de Educación en Ciencias de la Salud, 7 (2): 146-151. Recuperado de $\quad$ http://www2.udec.cl/ofem/recs/anteriores/vol722010/ revbib7210.htm

Pérez, A. F., Pérez, P., y García, J. A. (2016). Los objetivos generales y particulares en los programas educativos de las asignaturas UJAT: Un diagnóstico sintáctico y semántico. Perspectivas Docentes, 59, 28-34. Recuperado de http:/l revistas.ujat.mx/index.php/perspectivas/article/view/1306/1050

Pérez, A. F., Pérez, P., y Méndez, C. J. (2016). The learning outcomes in the study program format of school subjects belonging to the General Training Area at UJAT: A syntactic and semantic analysis. Perspectivas Docentes, 60, 15-20. Recuperado de http://revistas.ujat.mx/index.php/perspectivas/ article/view/1508/1318
Pontificia Universidad Javeriana. (2012). Estudio de seguimiento a recién egresados de programas académicos de pregrado de la Sede Central. Bogotá: PUJ. http://www. javeriana.edu.co/documents/15838/273636/SPEGRE2012V1. pdf/7ef592cf-da1d-4394-a134-b8a7ce8c3338

Romero, L. (2005). Profesionalización de la docencia universitaria: transformación y crisis. Impacto de la evaluación al desempeño de los académicos. Ciudad de México: UJATPlaza y Valdéz.

Universidad Juárez Autónoma de Tabasco. (2004). Programa institucional de seguimiento de egresados y opinión de empleadores. Villahermosa, Tabasco: UJAT.

Universidad Juárez Autónoma de Tabasco. (2005). Modelo educativo. Villahermosa, Tabasco: UJAT. Recuperado de http://www.archivos.ujat.mx/2011/dese/investigacion educativa2011/3 MODELO ED.pdf

Universidad Juárez Autónoma de Tabasco. (2006). Estudio de egresados en la licenciatura de médico cirujano (1997). Villahermosa, Tabasco: UJAT.

Universidad Juárez Autónoma de Tabasco. (2007). Estudio de empleadores. Villahermosa, Tabasco: UJAT.

Universidad Juárez Autónoma de Tabasco. (2010). Plan de estudios de la carrera de médico cirujano. Recuperado de http://www.archivos.ujat.mx/2013/damc/plan $\% 20$ medico $\% 20$ cirujano\%202010.pdf

Universidad Juárez Autónoma de Tabasco. (2012). Licenciatura en médico cirujano. En: Informe de seguimiento de egresados de la División Académica de Ciencias de la Salud, cohorte 2002. (pp. 158-208). Villahermosa: UJAT.

Watmough S.; O'Sullivan, H. y Taylor, D. (2009). Graduates from a traditional medical curriculum evaluate the effectiveness of their medical curriculum through interviews. BMC Medical Education, 9(64). doi: 10.1186/1472-6920-9-64 
Perspectivas Docentes 63

Percepción de pasantes de medicina en servicio social sobre su plan de estudios

Perception of graduates of the school of medicine in social service on its curriculum

Juan Manuel Muñoz Cano / Juan Antonio Córdova Hernández.

\section{SEMBLANZA DE LOS AUTORES}

Juan Manuel Muñoz Cano. Estudió medicina en la Universidad Juárez Autónoma de Tabasco (UJAT), donde es académico desde 1978. Especialista en Planeación y Administración de la Educación Superior, Maestro en Ciencias por el Programa de Biomedicina Molecular del Instituto Politécnico Nacional. Jefe del laboratorio 2 de biología molecular, de la UJAT. Ha escrito 15 libros, entre ellos "Alimentación humana: de la experiencia tradicional a la evidencia científica", "Cocina tradicional tabasqueña: componentes bioactivos, alimentos funcionales", en el campo de la difusión de la ciencia, y "El mundo de Tierraplana" y "El proyecto del Almirante" en el de la literatura.
Juan Antonio Córdova Hernández, Doctor en Educación. Maestría en Sistemas de Información, Perfil PRODEP, Miembro del Sistema Estatal de Investigadores, Profesor investigador de la Universidad Juárez Autónoma de Tabasco, División Académica de Ciencias de la Salud.

juan.cordova@ujat.mx

juan.munoz@ujat.mx 